\title{
An analytic study of the fractional order model of HIV-1 virus and CD4+ T-cells using adomian method
}

\author{
Kamel Al-Khaled, Maha Yousef \\ Department of Mathematics and Statistics, Jordan University of Science and Technology, Irbid, Jordan
}

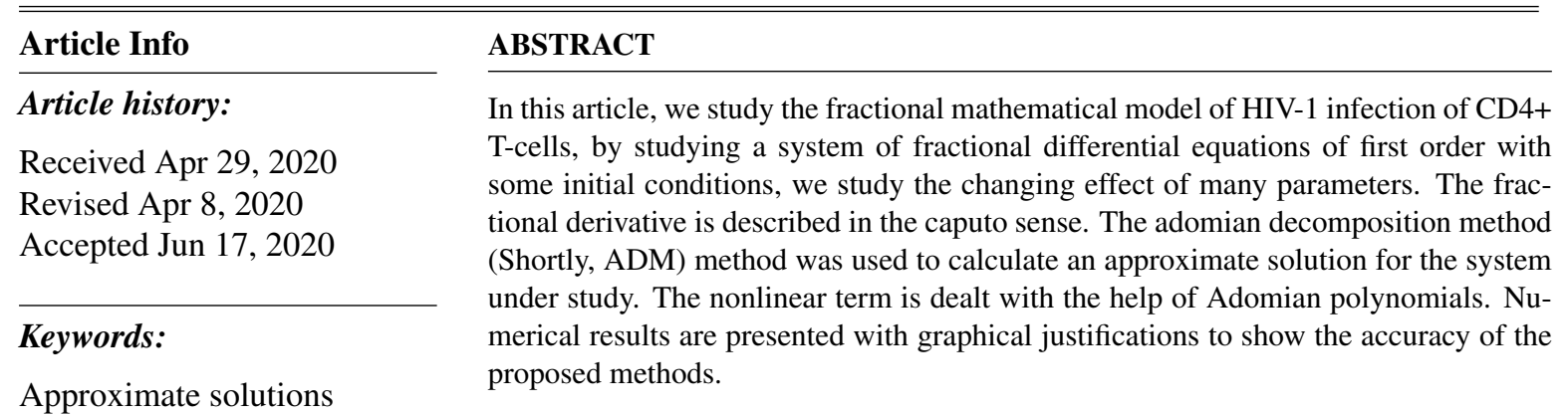

Fractional calculus

This is an open access article under the CC BY-SA license.

Adomian decomposition

Laplace transform

Fractional model for HIV

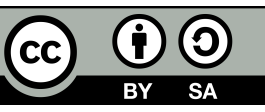

infection of CD4+ T-cells

Corresponding Author:

Kamel Al-Khaled

Jordan University of Science and Technology

Irbid, P.O.Box 3030, Jordan

Email: kamel@just.edu.jo

\section{INTRODUCTION}

There is a strong correlation coefficient between a person's immune system and an infection caused by virus, there there are many different factors and restrictions that affect this relationship, negatively or positively. When studying such a case, we cannot see the rules or foundations that control the dynamics or how infection occurs by studying the data or statistical drawings resulting from that study. By studying some mathematical models that simulate reality, we found that these models were important in dealing with a set of hypotheses related to the study through which the understanding of the study was reached in a logical and correct way. As a result of that, we can give find new hypotheses or scenarios which leads to the calculation of some numerical constants that have a significant impact in the study.

In this research, our main concern will be limited to the study of HIV. The study of the effect of dynamic processes such as movement and speed between the human immune system and HIV disease is a more complex fact compared to infection from other sides. The immune response then has the susceptibility and ability to fight the virus as HIV infects the auxiliary cells known as CD4+T [1]. It is one of the most abundant cells in the immune system, which controls the production of specific immune responses to the same goal. It must be noted here that HIV can affect some other immune cells that have an effective role in producing and generating anti-virus immunity. This means that the weakened immune response grows significantly and early during the first and advanced stage of the disease infection and this leads to viral tolerance and the ability to mutate and grow the virus. As for the development of AIDS, it occurs as a result of infection with the disease initially, the symptoms are not clear and for periods that may extend for several years, before the viral load increases and the number of cells called, CD4+ T-cells decreases to a low level, which leads to a decrease and weak resistance of the immune system in humans and as a result the disease spreads to the rest of the cells 
in the body. The development and spread of the disease depends on the development of some variables related to the virus itself, that is spreading quickly. There are some anti-retroviral therapies that have proven successful and have had a major impact in limiting the multiplication of the virus or postponing the development of the disease for long periods of time, this is proven true for many who have contracted the disease. These days, there are two ways to receive the medicine. The first is through reverse transcriptase inhibitors, where they overlap and prevent the infection of the healthy cell. As for the second mechanism, it prevents protease inhibitors from manufacturing a new infectious virus by the infected cell, meaning that the issue in general is either delaying time or Preventing the spread of the virus to prevent infection of healthy cells. Most of the infected cells have a relatively short lifespan, and therefore these cells live long. This means that eliminating the virus through medication is impossible. Taking medicines for this disease has several negative side effects, the most important of which is generating resistance against these drugs. This led to the researchers' keen interest in finding treatment regimens that strengthen the immune responses to the virus. Thus, thinking about a specific mechanism is to find therapeutic systems that support the immune responses to the virus.

Mathematical models have been devoted to understanding the transmission of HIV infection. There are many previous studies that were devoted to the study of transmission of HIV infection in humans, as these pedals were basically based on establishing a mathematical model, it is often made up of a system of ordinary differential equations with several parameters, researchers were able to find exact values for the parameters to use after solving the problem. Perelson, Kirschner and De Boer [2] they built a mathematical model that is a system of regular differential equations representing the spread of a virus that does not contain cells to HIV in a closed place that represents the bloodstream. The mathematical model consists of four main parts: the total number of healthy cells, then the total number of uninfected cells, the infected and infected cells with activity, and finally the free virus particles. This mathematical model is most popular for its HIV transmission. There are many related mathematical models that have been mentioned in the following previous studies [3-8], where the model of Perelson, Kirschner and De Boer, was used as a pillar in building these mathematical models. It has been observed that the mathematical model can be used to predict and know the symptoms of AIDS clinically, which is a good characteristic of the model. In the article [2], the researchers simplified the model that they proposed and wrote the model in the form of three differential differential equations of the first order through the hypothesis that all infected cells are the only ones capable of creating and producing cells infected with the virus. A mathematical model consisting of four equations was studied in a previous research by the first author and others [9] in that differential equations were used to be of ordinary derivatives without examining the fractional derivatives, that is new in this paper. Wang and Song in [10] found the equilibrium points of the mathematical system and study the stability of solutions that represent a cyclical solution to the model build for HIV. We can say that a large number of previous studies that dealt with the development of a mathematical model consisting of ordinary differential equations most of these studies were of the type that the derivatives are normal and not fractional order as in this research. In [11], Arafa studied an HIV model system of fractional order for CD+4 T-cells using the generalized euler method (GEM). Where in the study, the components were divided into three main parts, namely, the concentration of sensitive cells, the concentration of HIV-infected cells, and finally HIV particles that are denoted respectively by $T(t), I(t)$ and $V(t)$.

These days, fractional differentiation and integration has been extensively used in many fields with practical applications in science, engineering, and other knowledge [12-16]. Mathematicians were able to develop a fractional mathematical model related to the human root in [17]. Fractional derivatives embody the basic properties of cell rheological behavior and is highly successful in the subject that is related to rheology [18]. Talking about ordinary differential equations with fractional (FODE) derivatives is like talking about the memory that relates to that phenomenon in relation to biological systems [19]. In this paper, we will study a system of FODE which is a valid model for studying the development and growth of HIV, as we begin to define fractional derivatives and fractional integration. [20, 21]. Previous studies have shown that there are many definitions of fractional derivatives, and here only we mention the method that we will use which is Caputo's definition, which is suitable for solving differential equations with initial values.

The focus of this article is as, we present in section 2 the mathematical model under study related to HIV. In the third section, we briefly introduce some definitions of the fractional calculus. In section 4 , we extend the application of Adomian decomposition to build our analytical approximate solutions for the HIV-1 fractional system. Finally, graphical justification are displayed to validate the obtained solution. The paper ends with some concluding remarks. 


\section{FRACTIONAL DYNAMICAL MODEL HIV-1 INFECTION OF CD4+ T-CELLS}

Here we show the mathematical model of HIV virus infection of CD4+-cells in its general form with fractional derivatives. This model is written in the form of a system of regular non-linear differential equations that takes the following formulas.

$$
\left\{\begin{array}{l}
\frac{d^{\alpha} T}{d t^{\alpha}}=\delta-\mu T+r T\left(1-\frac{T+I}{T_{\max }}\right)-K V T \\
\frac{d^{\alpha} I}{d t^{\alpha}}=K V T-\beta I \\
\frac{d^{\alpha} V}{d t^{\alpha}}=N \beta I-\gamma V
\end{array} \quad, m-1<\alpha \leq m .\right.
$$

that has suitable conditions

$$
T(0)=T_{0}, I(0)=I_{0}, V(0)=v_{0}
$$

where $T(t), I(t)$ and $V(t)$ symbolizes to the concentration of sensitive CD4+ T-cells, the concentration of diseased CD4+ T-cells by the HIV viruses and the well HIV virus particles in the body, respectively. $r T(1-$ $\left.\frac{T+I}{T+a x}\right)$ is logistic model of the cell growth that are free of disease CD4 T-cells, and proliferation of infected CD4+ T-cells is ignored, $r$ is the rate of doubling the T-cells that are mitosis. $T_{\max }$ represent the largest stage of CD4+ T-cells in the infected body. The function $K V T$ represents an infected of HIV infection of diseasefree T-cells, here $K>0$ is the infection change. $N$ is the general rate of the number of disease-transmitting molecules that are produced from diseased cells throughout the entire cell life. The body is excreted CD4+ Tcells from precursors in the bone marrow and thymus at a fixed change of $\delta$. $\gamma$ per capita death rate of infected virus particles. $N \beta$ is the rate of production of virions by infected cells. It is noted that the set that follows represents some constraints with the appropriate constants for the aid of numerical solutions [2, 10].

$$
\begin{gathered}
T(0)=1.0 \times 10^{-1}, I(0)=0, V(0)=1.0 \times 10^{-1}, \mu=2.0 \times 10^{-2}, \beta=3.0 \times 10^{-1}, \\
\gamma=2.4, \delta=1.0 \times 10^{-1}, K=2.7 \times 10^{-3}, r=3, T_{\max }=1500, N=10 .
\end{gathered}
$$

\section{BASICS OF FRACTIONAL CALCULUS}

Fractional calculus and its applications encounter expeditious developments with more and more convincing applications in real world. Here we will present some of the characteristics related to the subject of fractional calculus where there are many definitions in previous studies, that have been proposed and we will mention here only the important part, that we will need to formulate the approximate solution to the issue that we study. These definitions include, Riemman-Liouville, Reize, Caputo, Rabotnov, Caputo-Fabrizio and Atangana-Baleanu fractional operator. The reason for finding fractional derivatives in relation to time is the characteristic representation of the kernel, in which each has its own difficulties, like the singularities that might accrues in the kernel, the memory effect caused by the locality of the kernel was major problem in the Caputo-Fabrizio fractional derivative was the best operator for dealing with systems involving the electroosmotic magneto hydrodynamic (MHD) free convectional flow of Walters'-B fluid in addition to the heat and mass produced [22]. Another fractional-exponential function is Yang-Abdel-Aty-Cattani FC, namely the YangAbdel-Aty-Cattani FC, is updated and addressed on propagation equations [23]. The main matter under study that is related to relation to fractional derivatives, is that we will re-present the material in section 2 of the research paper [24]. Here we will introduce the definition of Riemann-Liouville $J_{a}^{\alpha}$ as follows:

Definition 1 For a positive real number $\alpha$. The function $J^{\alpha}$, realized on the normal Lebesgue room $L_{1}[a, b]$ as

$$
J_{a}^{\alpha} f(x)=\frac{1}{\Gamma(\alpha)} \int_{a}^{x}(x-t)^{\alpha-1} f(t) d t, \quad J_{a}^{0} f(x)=f(x)
$$

when $a \leq x \leq b$, is purport to be the Riemann-Liouville fractional integral function with sort $\alpha$.

Some of the pairing properties of the function $J^{\alpha}$ can be found in the reference [14], and here we mention the important ones: For $f \in L_{1}[a, b], \alpha, \beta \geq 0$ and $\gamma>-1$

- $J_{a}^{\alpha} f(x)$ exists for almost every $x \in[a, b]$

$-J_{a}^{\alpha} J_{a}^{\beta} f(x)=J_{a}^{\alpha+\beta} f(x)$

- $J_{a}^{\alpha} J_{a}^{\beta} f(x)=J_{a}^{\beta} J_{a}^{\alpha} f(x)$ 
$-J_{a}^{\alpha} x^{\gamma}=\frac{\Gamma(\gamma+1)}{\Gamma(\alpha+\gamma+1)}(x-a)^{\alpha+\gamma}$

As seen in the reference [13], Riemann derivatives have some drawbacks when we solve realistic problems of fractional derivatives. Hence, we present an adjustment for the fractional differentiation function $D^{\alpha}$ presented by Caputo in his paper about the subject of visco-elasticity [25].

form

Definition 2 We define the fractional derivative of a given function $f(x)$ in the Caputo meaning in the

$$
D^{\alpha} f(x)=J^{m-\alpha} D^{m} f(x)=\frac{1}{\Gamma(m-\alpha)} \int_{0}^{x}(x-t)^{m-\alpha-1} f^{(m)}(t) d t,
$$

for values of $\alpha$ such that $m-1<\alpha \leq m$, here $m \in \mathbf{N}$, and $x>0$.

Here we mention two main characteristics, because we need them and we will use them later

Lemma 1 For alpha to satisfy $m-1<\alpha \leq m$, and the funtcion $\mathrm{f}$ to be in the class $L_{1}[a, b]$, we have $D_{a}^{\alpha} J_{a}^{\alpha} f(x)=f(x)$, also

$$
J_{a}^{\alpha} D_{a}^{\alpha} f(x)=f(x)-\sum_{k=0}^{m-1} f^{(k)}(0) \frac{(x-a)^{k}}{k !}, x>0 .
$$

One of the reasons for using the definition related to Caputo definition, is that in order to obtain a solution to the differential equation, additional conditions must be established in order to obtain a single unique solution. For the sense of having fractional differential equations of Caputo form, the mentioned supplementary conditions are just the usual constrains, that are taken to those of traditional equations. With comparison with Riemann-Liouville fractional differential equations, these supplementary terms give some fractional derivatives at the starting point $x=0$ of the solution to be found. Primitive conditions are not perceptible in addition to not knowing the amount of quantities to be calculated through the experiment, so that we can assign them perfectly in the analysis of the solution. There are many geometric and physical interpretations of fractional derivatives defined by the two methods Riemann-Liouville and Caputo types, it can be viewed by the reader in the research [13] due to its importance.

Definition 3 Let the number $m$, represent the smallest integer that is bigger than $\alpha$ by 1 , then the fractional derivatives of order $\alpha>0$, according to Caputa sense can be presented in the form

$$
D^{\alpha} u(x, t)=\frac{\partial^{\alpha} u(x, t)}{\partial t^{\alpha}}=\left\{\begin{array}{l}
\frac{1}{\Gamma(m-\alpha)} \int_{0}^{t}(t-\tau)^{m-\alpha-1} \frac{\partial^{m} u(x, \tau)}{\partial \tau^{m}} d \tau, \quad m-1<\alpha<m \\
\frac{\partial^{m} u(x, t)}{\partial t^{m}}, \quad \alpha=m \in N
\end{array}\right.
$$

For more information and to know the characteristics and proofs related to fractional derivatives, we recommend the reader to refer to the specialized references mentioned at the end of this research.

\section{IMPLEMENTATION OF ADOMIAN DECOMPOSITION METHOD}

In order to find our approximate solution for the nonlinear system of fractional differential in (1), via the use of the proposed methodology that is known as Adomian decomposition method. We assume that functions $T(t), I(t), V(t)$ are sufficiently differentiable and a unique solution for these equations exists.

Start by taking Laplace transform that is denoted by $\mathcal{L}$ of both sides of (1)

$$
\begin{array}{rlr}
\mathcal{L}\left\{\frac{d^{\alpha} T}{d t^{\alpha}}\right\} & =\mathcal{L}\{\delta\}-\mathcal{L}\{\mu T\}+\mathcal{L}\{r T\}-\frac{r}{T_{\max }} \mathcal{L}\left\{T^{2}\right\}-\frac{r}{T_{\max }} \mathcal{L}\{I T\}-\mathcal{L}\{K V T\}, \\
\mathcal{L}\left\{\frac{d^{\alpha} I}{d t^{\alpha}}\right\} & =\mathcal{L}\{K V T\}-\mathcal{L}\{\beta I\}, & 0<\alpha \leq 1 .
\end{array}
$$


Using the formulas of Laplace transform on the above equation

$$
\begin{array}{rlr}
s^{\alpha} \mathcal{L}\{T\}-T_{0} s^{\alpha-1} & =\frac{\delta}{s}-\mu \mathcal{L}\{T\}+r \mathcal{L}\{T\}-\frac{r}{T_{\max }} \mathcal{L}\left\{T^{2}\right\}-\frac{r}{T_{\max }} \mathcal{L}\{I T\}-K \mathcal{L}\{V T\}, \\
s^{\alpha} \mathcal{L}\{I\}-I_{0} s^{\alpha-1} & =K \mathcal{L}\{V T\}-\beta \mathcal{L}\{I\}, & 0<\alpha \leq 1 . \\
s^{\alpha} \mathcal{L}\{V\}-V_{0} s^{\alpha-1} & =N \beta \mathcal{L}\{I\}-\gamma \mathcal{L}\{V\}, &
\end{array}
$$

Substituting the initial conditions, we arrive at

$$
\begin{array}{rlrl}
s^{\alpha} \mathcal{L}\{T\} & =0.1 s^{\alpha-1}+\frac{\delta}{s}-\mu \mathcal{L}\{T\}+r \mathcal{L}\{T\}-\frac{r}{T_{\max }} \mathcal{L}\left\{T^{2}\right\}-\frac{r}{T_{\max }} \mathcal{L}\{I T\}-K \mathcal{L}\{V T\}, \\
s^{\alpha} \mathcal{L}\{I\} & =K \mathcal{L}\{V T\}-\beta \mathcal{L}\{I\}, & 0<\alpha \leq 1 .
\end{array}
$$

or

$$
\begin{aligned}
\mathcal{L}\{T\} & =\frac{0.1}{s}+\frac{\delta}{s^{\alpha+1}}-\frac{\mu}{s^{\alpha}} \mathcal{L}\{T\}+\frac{r}{s^{\alpha}} \mathcal{L}\{T\}-\frac{r}{s^{\alpha} T_{\max }} \mathcal{L}\left\{T^{2}\right\}-\frac{r}{s^{\alpha} T_{\max }} \mathcal{L}\{I T\}-\frac{K}{s^{\alpha}} \mathcal{L}\{V T\} \\
\mathcal{L}\{I\} & =\frac{K}{s^{\alpha}} \mathcal{L}\{V T\}-\frac{\beta}{s^{\alpha}} \mathcal{L}\{I\}, \\
\mathcal{L}\{V\} & =\frac{0.1}{s}+\frac{N \beta}{s^{\alpha}} \mathcal{L}\{I\}-\frac{\gamma}{s^{\alpha}} \mathcal{L}\{V\},
\end{aligned}
$$

To obtain the approximate solution, we follow the Adomian's method, which depends on writing the solution as an infinite bounded series, according to the following:

$$
T(t)=\sum_{n=0}^{\infty} T_{n}(t), \quad I(t)=\sum_{n=0}^{\infty} I_{n}(t), \quad V(t)=\sum_{n=0}^{\infty} V_{n}(t),
$$

which will be determined recursively according to a recursive relation. Moreover, to overcome the non linearity problem, the method defines the nonlinear functions $A=T^{2}, B=T I, C=V T$ by an infinite sum of polynomials, defined by

$$
A=\sum_{n=0}^{\infty} A_{n}, \quad B=\sum_{n=0}^{\infty} B_{n}, \quad C=\sum_{n=0}^{\infty} C_{n}
$$

where the $A_{n}, B_{n}, C_{n}$ are the Adomian's polynomials which are generated according to some formulas set by Adomian [26, 27], or as in the modified form done by Wazwaz [28].

$$
\begin{aligned}
& A_{0}=T_{0}^{2}, \\
& A_{1}=2 T_{0} T_{1}, \\
& A_{2}=2 T_{0} T_{2}+T_{1}^{2}, \\
& A_{3}=2 T_{0} T_{3}+2 T_{1} T_{2}, \\
& A_{4}=2 T_{0} T_{4}+2 T_{1} T_{3}+\left(T_{2}\right)^{2}, \\
& B_{0}=T_{0} I_{0}, \\
& B_{1}=T_{0} I_{1}+T_{1} I_{0}, \\
& B_{3}=T_{0} I_{3}+T_{1} I_{2}+T_{2} I_{1}+T_{3} I_{0}, \\
& B_{4}=T_{0} I_{4}+T_{1} I_{3}+T_{2} I_{2}+T_{3} I_{1}+T_{4} I_{0}, \\
& C_{0}=V_{0} T_{1}+V_{1} T_{0}, \\
& C_{1}=V_{0} T_{1}+V_{1} T_{0}, \\
& C_{3}=V_{0} T_{3}+V_{1} T_{2}+V_{2} T_{1}+V_{3} T_{0}, \\
& C_{4}=V_{0} T_{4}+V_{1} T_{3}+V_{2} T_{2}+V_{3} T_{1}+V_{4} T_{0},
\end{aligned}
$$


Substituting (5) and (6) into (4) yields

$$
\begin{aligned}
& \mathcal{L}\left\{\sum_{n=0}^{\infty} T_{n}(t)\right\}=\frac{0.1}{s}+\frac{\delta}{s^{\alpha+1}}-\frac{\mu}{s^{\alpha}} \mathcal{L}\left\{\sum_{n=0}^{\infty} T_{n}(t)\right\}+\frac{r}{s^{\alpha}} \mathcal{L}\left\{\sum_{n=0}^{\infty} T_{n}(t)\right\} \\
&-\frac{r}{s^{\alpha} T_{\max }} \mathcal{L}\left\{\sum_{n=0}^{\infty} A_{n}(t)\right\}-\frac{r}{s^{\alpha} T_{\max }} \mathcal{L}\left\{\sum_{n=0}^{\infty} B_{n}(t)\right\}-\frac{K}{s^{\alpha}} \mathcal{L}\left\{\sum_{n=0}^{\infty} C_{n}(t)\right\}, \\
& \mathcal{L}\left\{\sum_{n=0}^{\infty} I_{n}(t)\right\} \quad=\frac{K}{s^{\alpha}} \mathcal{L}\left\{\sum_{n=0}^{\infty} C_{n}(t)\right\}-\frac{\beta}{s^{\alpha}} \mathcal{L}\left\{\sum_{n=0}^{\infty} I_{n}(t)\right\}, \\
& \mathcal{L}\left\{\sum_{n=0}^{\infty} V_{n}(t)\right\}=\frac{0.1}{s}+\frac{N \beta}{s^{\alpha}} \mathcal{L}\left\{\sum_{n=0}^{\infty} I_{n}(t)\right\}-\frac{\gamma}{s^{\alpha}} \mathcal{L}\left\{\sum_{n=0}^{\infty} V_{n}(t)\right\},
\end{aligned}
$$

It is to be observed that the iterative relation is constructed on the basis that the first iteration of the solution $\mathcal{L}\left[T_{0}\right], \mathcal{L}\left[I_{0}\right]$, and $\mathcal{L}\left[V_{0}\right]$ are obtained those values that generated from the initial conditions, i.e.,

$$
\begin{aligned}
\mathcal{L}\left\{T_{0}(t)\right\} & =\frac{0.1}{s}+\frac{\delta}{s^{\alpha+1}}, \\
\mathcal{L}\left\{T_{1}(t)\right\} & =-\frac{\mu}{s^{\alpha}} \mathcal{L}\left\{T_{0}(t)\right\}+\frac{r}{s^{\alpha}} \mathcal{L}\left\{T_{0}(t)\right\}-\frac{r}{s^{\alpha} T_{\max }} \mathcal{L}\left\{A_{0}(t)\right\}-\frac{r}{s^{\alpha} T_{\max }} \mathcal{L}\left\{B_{0}(t)\right\}-\frac{K}{s^{\alpha}} \mathcal{L}\left\{C_{0}(t)\right\}, \\
\mathcal{L}\left\{T_{2}(t)\right\} & =-\frac{\mu}{s^{\alpha}} \mathcal{L}\left\{T_{1}(t)\right\}+\frac{r}{s^{\alpha}} \mathcal{L}\left\{T_{1}(t)\right\}-\frac{r}{s^{\alpha} T_{\max }} \mathcal{L}\left\{A_{1}(t)\right\}-\frac{r}{s^{\alpha} T_{\max }} \mathcal{L}\left\{B_{1}(t)\right\}-\frac{K}{s^{\alpha}} \mathcal{L}\left\{C_{1}(t)\right\}, \\
\mathcal{L}\left\{T_{n+1}(t)\right\} & =-\frac{\mu}{s^{\alpha}} \mathcal{L}\left\{T_{n}(t)\right\}+\frac{r}{s^{\alpha}} \mathcal{L}\left\{T_{n}(t)\right\}-\frac{r}{s^{\alpha} T_{\max }} \mathcal{L}\left\{A_{n}(t)\right\}-\frac{r}{s^{\alpha} T_{\text {max }}} \mathcal{L}\left\{B_{n}(t)\right\}-\frac{K}{s^{\alpha}} \mathcal{L}\left\{C_{n}(t)\right\}, \\
\mathcal{L}\left\{I_{0}(t)\right\} & =0, \\
\mathcal{L}\left\{I_{1}(t)\right\} & =\frac{K}{s^{\alpha}} \mathcal{L}\left\{C_{0}(t)\right\}-\frac{\beta}{s^{\alpha}} \mathcal{L}\left\{I_{0}(t)\right\}, \\
\mathcal{L}\left\{I_{2}(t)\right\} & =\frac{K}{s^{\alpha}} \mathcal{L}\left\{C_{1}(t)\right\}-\frac{\beta}{s^{\alpha}} \mathcal{L}\left\{I_{1}(t)\right\}, \\
\mathcal{L}\left\{I_{n+1}(t)\right\} & =\frac{K}{s^{\alpha}} \mathcal{L}\left\{C_{n}(t)\right\}-\frac{\beta}{s^{\alpha}} \mathcal{L}\left\{I_{n}(t)\right\}, \\
\mathcal{L}\left\{V_{0}(t)\right\} & =\frac{0.1}{s}, \\
\mathcal{L}\left\{V_{1}(t)\right\} & =\frac{N \beta}{s^{\alpha}} \mathcal{L}\left\{I_{0}(t)\right\}-\frac{\gamma}{s^{\alpha}} \mathcal{L}\left\{V_{0}(t)\right\}, \\
\mathcal{L}\left\{V_{2}(t)\right\} & =\frac{N \beta}{s^{\alpha}} \mathcal{L}\left\{I_{1}(t)\right\}-\frac{\gamma}{s^{\alpha}} \mathcal{L}\left\{V_{1}(t)\right\}, \\
\mathcal{L}\left\{V_{n+1}(t)\right\} & =\frac{N \beta}{s^{\alpha}} \mathcal{L}\left\{I_{n}(t)\right\}-\frac{\gamma}{s^{\alpha}} \mathcal{L}\left\{V_{n}(t)\right\},
\end{aligned}
$$

As a result, the components are identified by applying inverse Laplace transform of the above equations, to obtain

$$
\begin{aligned}
& T_{0}(t)=0.1+\mathcal{L}^{-1}\left\{\frac{\delta}{s^{\alpha+1}}\right\} \\
& I_{0}(t)=0 \\
& V_{0}(t)=0.1
\end{aligned}
$$


and

$$
\begin{aligned}
T_{n+1}(t) & =\mathcal{L}^{-1}\left\{\frac{-\mu}{s^{\alpha}} \mathcal{L}\left\{T_{n}(t)\right\}+\frac{r}{s^{\alpha}} \mathcal{L}\left\{T_{n}(t)\right\}-\frac{r}{s^{\alpha} T_{\max }} \mathcal{L}\left\{A_{n}(t)\right\}-\frac{r}{s^{\alpha} T_{\max }} \mathcal{L}\left\{B_{n}(t)\right\}-\frac{K}{s^{\alpha}} \mathcal{L}\left\{C_{n}(t)\right\},\right\} \\
I_{n+1}(t) & =\mathcal{L}^{-1}\left\{\frac{K}{s^{\alpha}} \mathcal{L}\left\{C_{n}(t)\right\}-\frac{\beta}{s^{\alpha}} \mathcal{L}\left\{I_{n}(t)\right\}\right\}, \\
V_{n+1}(t) & =\mathcal{L}^{-1}\left\{\frac{N \beta}{s^{\alpha}} \mathcal{L}\left\{I_{n}(t)\right\}-\frac{\gamma}{s^{\alpha}} \mathcal{L}\left\{V_{n}(t)\right\}\right\},
\end{aligned}
$$

Thus, the solutions in the form of an infinite series are entirely founded. However in many cases (especially when $\alpha$ is an integer) the exact solution in a more compact form may be obtained. The $n-t h$ term approximation $\Phi_{n}=\sum_{k=0}^{n-1} T_{k}, \Psi_{n}=\sum_{k=0}^{n-1} I_{k}, \Theta_{n}=\sum_{k=0}^{n-1} V_{k}$ can be used to approximate the solution. The way we choose the initial solution always leads to noise oscillation during the iteration technique. In addition, the choice of $T_{0}(t), I_{0}(t), V_{0}(t)$ containing few number of terms gives more flexibility to solve complicated nonlinear equations, especially when evaluating the inverse of the laplace transform. A trustworthy alter form of the adomian's method has been mentioned and discussed by Wazwaz [28]. The construction of the zeroth term of the decomposition series can be defined by splitting the zeroth term into the sum of two terms, then the first term assigned to $T_{0}(t)$, while the other remaining term for $T_{0}(t)$ be assigned to the second component $T_{1}(t)$. To obtain the inverse laplace tranform of (13), we perform Mathematica simple calculations. The approximate solution is given by $T(t)=\sum_{k=0}^{3} T_{k}, I(t)=\sum_{k=0}^{3} I_{k}$ and $V(t)=\sum_{k=0}^{3} V_{k}$. The numerical results shown in Figures 1 and 2 imply the efficiency of the proposed method discussed here. This method gives highly accurate results in very few iterations.

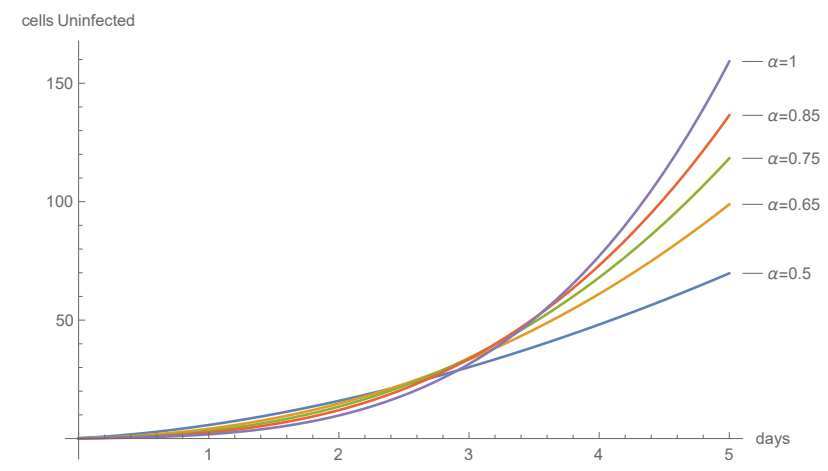

Figure 1. Dynamics of uninfected CD4+ T-cells for various values of $\alpha$

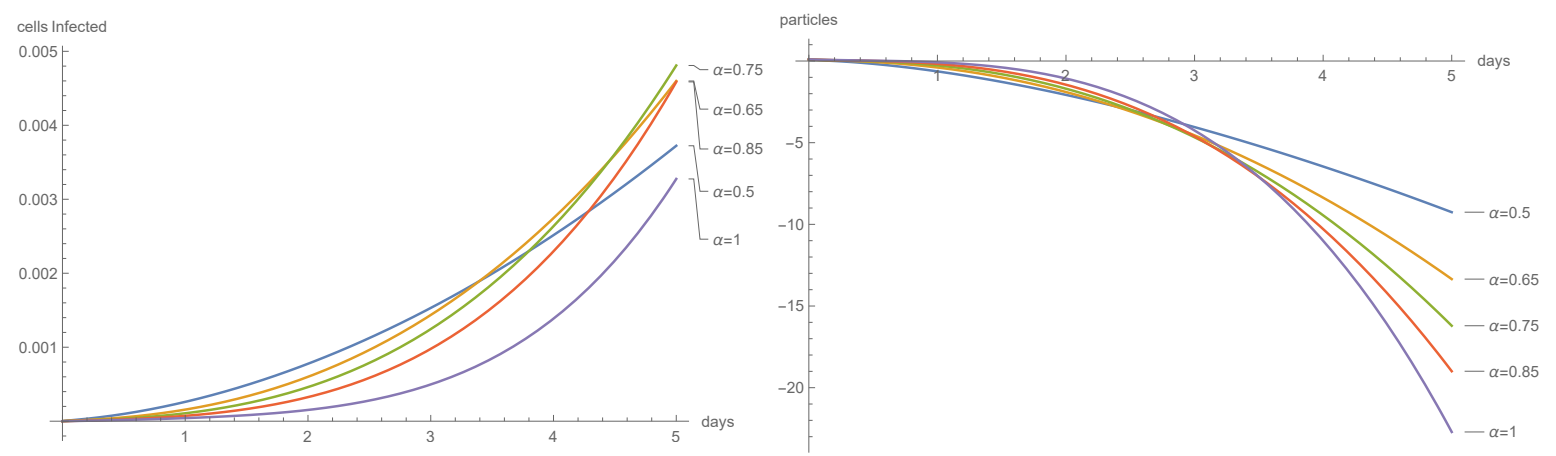

Figure 2. Dynamics of infected CD4+ T-cells and dynamics of density of particles in plasma for various values of $\alpha$ 


\section{CONCLUSION}

The elementary goal of this paper is to suggest a systematic algorithm for the solution of fractional ordinary differential system of HIV-1 virus and CD4+ T-Cells . This goal has been achieved by using adomian decomposition method with the successful help of laplace tranform. The validity and accuracy of our approach is examined by focusing on the changing behavior of $\alpha$ in the previous illustrative plots that guarantee that the adomian decomposition method is very helpful and effective method to produce analytical solutions. Finally, The above method can solve the nonlinear cases with no difficulty, without linearization, perturbation or discretization.

\section{ACKNOWLEDGEMENT}

This research was supported by deanship of research at Jordan University of Science and Technology (JUST), Grant No. 168-2019. We thank our University (JUST) and colleagues who provided insight and expertise that greatly assisted the research.

\section{REFERENCES}

[1] R.V. Culshaw, S. Ruan., "A delay-differential equation model of HIV infection of CD4C T-cells," Mathematical Bioscience, vol. 165, no. 1, pp. 27-39, 2000, doi: 10.1016/s0025-5564(00)00006-7.

[2] A.S. Perelson, D.E. Kirschner, R. De Boer., "Dynamics of HIV infection of CD4C T-cells, Mathematical Bioscience," vol. 114, no. 1, pp. 81-125,1993, doi: 10.1016/0025-5564(93)90043-a.

[3] Lichae BH., Biazar J., Ayati Z., "The fractional differential model of HIV-1 infection of CD4+ T-cells with description of the effect of antiviral drug treatment," Computational and Mathematical Methods in Medicine, 2019, https://doi.org/10.1155/2019/4059549.

[4] Kumar S., Kumar R., Singh J., Nisar KS., Kumar D., "An efficient numerical scheme for fractional model of HIV-1 infection of CD4+ T-cells with the effect of antiviral drug therapy," Alexandria Engineering Journal, vol. 59, no. 4, pp. 2053-2064, 2020, https://doi.org/10.1016/j.aej.2019.12.046.

[5] Ahmed, E., El-Sayed, A. M. A., and El-Saka, H. A., "Equilibrium points, stability and numerical solutions of fractional-order predator-prey and rabies models," Journal of Mathematical Analysis and Applications, vol. 325, no. 1, 542-553, 2007.

[6] Al-Khaled, K., "Sinc-Galerkin Method for Solving Nonlinear Fractional Fourth-Order Boundary Value Problems," In Proceedings of International Conference on Fractional Differentiation and its Applications (ICFDA), July, 2018.

[7] I. Podlubny, "Fractional Differential Equations," Academic Press, New York, 1999.

[8] R. Hilfer (Ed.), "Applications of fractional calculus in physics," World Scientific Pub Co., Singapore, 2000.

[9] Alquran M., Al-Khaled K., Alawneh A., "Simulated results for deterministic model of HIV dynamics," Stud. Univ. Babes-Bolyai Math., vol.56, no. 1, pp. 165-78, 2011.

[10] Wang, Xia, and Xinyu Song., "Global stability and periodic solution of a model for HIV infection of CD4+ T cells," Applied Mathematics and Computation, vol. 189, no. 2, pp. 1331-1340, 2007, doi: https://doi.org/10.1016/j.amc.2006.12.044.

[11] Arafa, A. A. M., Rida, S. Z.,and Khalil, M., "Fractional modeling dynamics of HIV and CD4+ T-cells during primary infection," Nonlinear biomedical physics, vol. 6, no. 1, 2012, doi: 10.1186/1753-4631-6-1.

[12] Jesus, I. S., Tenreiro Machado, J. A., Cunha, J. B., and Silva, M., "Fractional order electrical impedance of fruits and vegetables," In 25th IASTED International Conference Modelling, Identification and Control, 2006, pp. 489-494.

[13] K. B. Oldham, J. Spanier., "The Fractional Calculus," Academic Press, New York, 1974.

[14] I. Podlubny, "Geometric and physical interpretation of fractional integration and fractional differentiation," Frac. Calc. Appl. Anal., vol. 5, no. 4, pp. 367-386, 2002.

[15] Ongun, M. Y., "The Laplace Adomian decomposition method for solving a model for HIV infection of CD4+ T cells," Mathematical and Computer Modelling, vol. 53, no. 5-6, pp. 597-603, 2011.

[16] Agraj Tripathi, Ram Naresh and Dileep Sharma, "Modeling the effect of screening of unaware infectives on the spread of HIV infection," Applied Mathematics and Computation, vol. 184, no. 2, pp. 1053-1068, 2007. 
[17] Rossikhin, Yury A., and Marina V. Shitikova, "Analysis of two colliding fractionally damped spherical shells in modelling blunt human head impacts," Central European Journal of Physics, vol. 11, pp. 760-778, 2013.

[18] V.D. Djordjevic, J. Jaric, B. Fabry, J.J. Fredberg, D. Stamenovic, "Fractional derivatives embody essential features of cell rheological behavior," Annals of Biomedical Engineering vol. 31, pp. 692-699, 2003.

[19] Ahmed N., Ali M., Rafiq M., Khan I., Nisar KS, Rehman MA., Ahmad MO., "A numerical efficient splitting method for the solution of two dimensional susceptible infected recovered epidemic model of whooping cough dynamics: Applications in bio-medical engineering," Computer Methods and Programs in Biomedicine, vol. 190, 2020, doi: https://doi.org/10.1016/j.cmpb.2020.105350.

[20] Al-Khaled K., "Sinc collocation linked with finite differences for Korteweg-de Vries Fractional Equation," International Journal of Electrical and Computer Engineering (IJECE), vol. 10, no. 1, pp. 512-520, 2020.

[21] Al-Khaled K., Abu-Irwaq I., "Computational Sinc-scheme for extracting analytical solution for the model Kuramoto-Sivashinsky equation," International Journal of Electrical and Computer Engineering (IJECE), vol. 9, no. 5, pp. 3720-3731, 2019

[22] Ali F., Iftikhar M., Khan I., Sheikh NA, Nisar KS., "Time fractional analysis of electro-osmotic flow of Walters' sB fluid with time-dependent temperature and concentration," Alexandria Engineering Journal, vol. 59, no. 1, pp. 25-38, 2020.

[23] Kumar S., Nisar KS, Kumar R., Cattani C., Samet B., "A new Rabotnov fractional-exponential functionbased fractional derivative for diffusion equation under external force," Mathematical Methods in the Applied Sciences, vol. 43, no. 7, pp. 4460-71, 2020.

[24] Al-Khaled K., "Numerical solution of time-fractional partial differential equations using Sumudu decomposition method," Rom. J. Phys., vol. 60, no. 1-2, pp. 99-110, 2015.

[25] Caputo M., "Linear models of dissipation whose Q is almost frequency independent- II," Geophys. J. R. Astron. Soc., vol. 13, no. 5, pp. 529-539, 1967, doi: https://doi.org/10.1111/j.1365-246X.1967.tb02303.x.

[26] G. Adomian, "A review of the decomposition method in applied Mathematics," J. Math. Anal. Appl. vol. 135, no. 2, pp. 501-544, 1988, doi: https://doi.org/10.1016/0022-247X(88)90170-9.

[27] G. Adomian, "Solving Frontier problems of physics: The Decomposition Method," Kluwer, Boston, 1994.

[28] Wazwaz AM, "A new algorithm for calculating Adomian polynomials for nonlinear operators," Applied Mathematics and computation," vol. 111, no. 1, pp. 33-51, 2000. 